\title{
THE STRONG LIMIT OF VON NEUMANN SUBALGEBRAS WITH CONDITIONAL EXPECTATIONS ${ }^{1}$
}

\author{
MAKOTO TSUKADA
}

\begin{abstract}
The strong lower limit and the weak upper limit of a net of von Neumann subalgebras on which the conditional expectations exist with respect to a fixed faithful normal state are defined. The limits coincide if and only if the corresponding conditional expectations converge strongly.
\end{abstract}

1. Preliminaries. Let $M$ be a $\sigma$-finite von Neumann algebra and $\varphi$ a faithful normal state on $M$. By the GNS construction it can be considered that $M$ is acting on a Hilbert space $H$ and there exists a cyclic separating vector $\Phi \in H$ with $\varphi(x)=\langle\Phi \mid x \Phi\rangle$ for every $x \in M$. Denote by $M_{*}$ the space of all $\sigma$-weakly continuous linear functionals on $M$. That is, $M_{*}$ is the predual of $M$.

For a von Neumann subalgebra $N$ of $M$, if there exists a projection $\varepsilon$ of norm one from $M$ onto $N$ with $\varphi \circ \varepsilon=\varphi, \varepsilon$ is called the conditional expectation onto $N[3,6]$.

$1^{\circ}$. The conditional expectation onto $N$ exists if and only if $\sigma_{t}(N)=N$ for every $t \in \mathbf{R}$, where $\left\{\sigma_{t}\right\}$ is the modular automorphism group on $M$ with respect to $\varphi$.

$2^{\circ}$. If the conditional expectation $\varepsilon$ onto $N$ exists, then $\varepsilon(x) \Phi=P x \Phi$ for every $x \in M$, where $P$ is the orthogonal projection of $H$ onto $\overline{N \Phi}$.

Throughout this paper we fix a net $\left\{N_{\alpha}\right\}$ of von Neumann subalgebras of $M$ and assume that the conditional expectation $\varepsilon_{\alpha}$ onto $N_{\alpha}$ exists for each $\alpha$. The orthogonal projection of $H$ onto $H_{\alpha}=\widehat{N_{\alpha} \Phi}$ is denoted by $P_{\alpha}$. In the recent paper [5] we proved that if $\left\{N_{\alpha}\right\}$ is increasing (resp. decreasing), then the conditional expectation $\varepsilon_{\infty}$ onto $\bigvee_{\alpha} N_{\alpha}$ (resp. $\bigcap_{\alpha} N_{\alpha}$ ) exists and $\varepsilon_{\alpha}(x) \rightarrow \varepsilon_{\infty}(x)$ strongly for every $x \in M$ and $f \circ \varepsilon_{\alpha} \rightarrow f \circ \varepsilon_{\infty}$ in norm for every $f \in M_{*}$. In this paper we shall introduce the notion of the strong limit of $\left\{N_{\alpha}\right\}$ and show that the limit exists if and only if the corresponding $\left\{\varepsilon_{\alpha}\right\}$ converge strongly. The following are elementary but will be useful below.

$3^{\circ}$. For any uniformly bounded net $\left\{x_{\gamma}\right\}$ in $M$ and $x \in M, x_{\gamma} \rightarrow x$ strongly (resp. weakly) if and only if $x_{\gamma} \Phi \rightarrow x \Phi$ strongly (resp. weakly) in $H$.

$4^{\circ}$. Let $\left\{P_{\gamma}\right\}$ be a net of orthogonal projections of $H$, and $P$ an orthogonal projection of $H$. For any $\xi \in H$, if $P_{\gamma} \xi \rightarrow P \xi$ weakly, then it does strongly.

Received by the editors January 23, 1984 and, in revised form. July 16, 1984.

1980 Mathematics Subject Classification. Primary 46L10.

Kel n'ords and phrases. Faithful normal state, conditional expectation, martingale.

'This work is part of the author's doctoral dissertation written under the advice of $\mathrm{H}$. Umegaki and presented to Tokyo Institute of Technology. 
2. The strong limit of $\left\{N_{\alpha}\right\}$. We define the strong lower limit $s$-liminf $N_{\alpha}$ and the weak upper limit $w$-lim $\sup N_{\alpha}$ of $\left\{N_{\alpha}\right\}$ as follows:

I. $x \in s$-liminf $N_{\alpha}$ if and only if there exist $x_{\alpha} \in N_{\alpha}$ for each $\alpha$ such that $\sup _{\alpha}\left\|x_{\alpha}\right\|<\infty$ and $x_{\alpha} \rightarrow x$ strongly;

II. $x \in w$-lim sup $N_{\alpha}$ if and only if there exist $x_{\alpha^{\prime}} \in N_{\alpha^{\prime}}$ for each $\alpha^{\prime}$, where $\left\{N_{\alpha^{\prime}}\right\}$ is a subnet of $\left\{N_{\alpha}\right\}$, such that $\sup _{\alpha^{\prime}}\left\|x_{\alpha^{\prime}}\right\|<\infty$ and $x_{\alpha^{\prime}} \rightarrow x$ weakly.

THEOREM 1. (i) s-liminf $N_{\alpha}$ is a von Neumann subalgebra of $M$ and satisfies the following equalities:

$$
\begin{aligned}
s-\lim \inf N_{\alpha} & =\left\{x \in M: \varepsilon_{\alpha}(x) \rightarrow x \text { strongly }\right\} \\
& =\left\{x \in M: \varepsilon_{\alpha}(x) \rightarrow x \text { weakly }\right\} .
\end{aligned}
$$

(ii) Both the conditional expectation onto $s$-liminf $N_{\alpha}$ and the one onto $\left(w-\lim \sup N_{\alpha}\right)^{\prime \prime}$ exist.

$$
\bigvee_{\beta} \bigcap_{\alpha \geqslant \beta} N_{\alpha} \subseteq s-\lim \inf N_{\alpha} \subseteq w-\lim \sup N_{\alpha} \subseteq \bigcap_{\beta} \bigvee_{\alpha \geqslant \beta} N_{\alpha}
$$

Proof. (i) We establish the first equality. " $\supseteq$ " is clear. Let $x$ belong to $s$-liminf $N_{\alpha}$. There exists a uniformly bounded net $\left\{x_{\alpha}\right\}$ such that $x_{\alpha} \in N_{\alpha}$ for each $\alpha$ and $x_{\alpha} \rightarrow x$ strongly. Then

$$
\left\|\varepsilon_{\alpha}(x) \Phi-x \Phi\right\|=\left\|P_{\alpha} x \Phi-x \Phi\right\| \leqslant\left\|x_{\alpha} \Phi-x \Phi\right\| \rightarrow 0 \quad(\text { as } \alpha \uparrow) .
$$

Thus $\varepsilon_{\alpha}(x) \rightarrow x$ strongly by $3^{\circ}$ in $\S 1$. The second equality follows from $2^{\circ}, 3^{\circ}$ and $4^{\circ}$ in $\S 1$. We next show that $s$-lim inf $N_{\alpha}$ is a von Neumann subalgebra of $M$. It is clearly closed under linear combination. Let $x$ and $y$ belong to $s$-lim inf $N_{\alpha}$. Then

$$
\begin{aligned}
& \left\|\varepsilon_{\alpha}(x) \varepsilon_{\alpha}(y) \Phi-x y \Phi\right\| \\
& \quad \leqslant\left\|\varepsilon_{\alpha}(x) \varepsilon_{\alpha}(y) \Phi-\varepsilon_{\alpha}(x) y \Phi\right\|+\left\|\varepsilon_{\alpha}(x) y \Phi-x y \Phi\right\| \\
& \quad \leqslant\|x\| \cdot\left\|\varepsilon_{\alpha}(y) \Phi-y \Phi\right\|+\left\|\varepsilon_{\alpha}(x) y \Phi-x y \Phi\right\| \rightarrow 0 \quad(\text { as } \alpha \uparrow) .
\end{aligned}
$$

Hence $x y \in s$-lim inf $N_{\alpha}$. On the other hand, since $\varepsilon_{\alpha}(x) \rightarrow x$ weakly, $\varepsilon_{\alpha}\left(x^{*}\right) \rightarrow x^{*}$ weakly. Therefore $x^{*} \in s$-liminf $N_{\alpha}$. Finally, let $x$ belong to the strong closure of $s-\lim \inf N_{\alpha}$. Then for any $\varepsilon>0$ there exists $y \in s-\lim \inf N_{\alpha}$ such that $\|x \Phi-y \Phi\|<$ $\varepsilon / 3$, and for this $y$ there exists $\alpha_{0}$ such that $\left\|\varepsilon_{\alpha}(y) \Phi-y \Phi\right\|<\varepsilon / 3$ for any $\alpha \geqslant \alpha_{0}$. Hence

$$
\begin{aligned}
\left\|\varepsilon_{\alpha}(x) \Phi-x \Phi\right\| & \leqslant\left\|P_{\alpha} x \Phi-P_{\alpha} y \Phi\right\|+\left\|P_{\alpha} y \Phi-y \Phi\right\|+\|y \Phi-x \Phi\| \\
& \leqslant 2 \cdot\|x \Phi-y \Phi\|+\left\|\varepsilon_{\alpha}(y) \Phi-y \Phi\right\|<\varepsilon
\end{aligned}
$$

for any $\alpha \geqslant \alpha_{0}$. Therefore $\varepsilon_{\alpha}(x) \rightarrow x$ strongly and we have $x \in s$-liminf $N_{\alpha}$.

(ii) This follows from $1^{\circ}$ in $\S 1$. Indeed, let $x \in s$-liminf $N_{\alpha}$. Then there exist $x_{\alpha} \in N_{\alpha}$ for each $\alpha$ such that $x_{\alpha} \rightarrow x$ strongly. Since $\sigma_{t}\left(x_{\alpha}\right) \in N_{\alpha}$ for each $\alpha$ and $\sigma_{t}\left(x_{\alpha}\right) \rightarrow \sigma_{t}(x)$ strongly, $\sigma_{t}(x) \in s$-liminf $N_{\alpha}$. Thus $s$-liminf $N_{\alpha}$ is globally invariant under the modular automorphism group. Similarly, we have

$$
\sigma_{t}\left(w-\lim \sup N_{\alpha}\right)=w-\lim \sup N_{\alpha} .
$$


Since $\sigma_{t}$ is an automorphism,

$$
\sigma_{t}\left(w-\lim \sup N_{\alpha}\right)^{\prime \prime}=\sigma_{t}\left(\left(w-\lim \sup N_{\alpha}\right)^{\prime \prime}\right) .
$$

Thus ( $w$-lim $\left.\sup N_{\alpha}\right)$ " is also globally invariant under the automorphism group.

(iii) This is easily verified.

EXAmples. (i) Let $N_{0}$ and $N_{1}$ be von Neumann subalgebras with conditional expectations and $N_{2 n}=N_{0}$ and $N_{2 n+1}=N_{1}$ for $n=1,2, \ldots$ Then $s-\lim \inf N_{n}=N_{0}$ $\cap N_{1}$ and $w$-limsup $N_{n}=N_{0} \cup N_{1}$. Hence, in general, $s-\lim \inf N_{n} \neq w-\lim \sup N_{n}$ and $w$-lim sup $N_{n}$ is not a von Neumann subalgebra.

(ii) If $\left\{N_{\alpha}\right\}$ is increasing (resp. decreasing) and $N_{\infty}=\mathrm{V}_{\alpha} N_{\alpha}$ (resp. $\bigcap_{\alpha} N_{\alpha}$ ), then $\mathrm{V}_{\beta} \bigcap_{\alpha \geqslant \beta} N_{\alpha}=\bigcap_{\beta} \mathrm{V}_{\alpha \geqslant \beta} N_{\alpha}=N_{\infty}$. In this case by Theorem 1 (iii),

$$
s-\lim \inf N_{\alpha}=w \text {-lim } \sup N_{\alpha}=N_{\infty} \text {. }
$$

(iii) Denote by $\operatorname{Aut}_{\varphi}(M)$ the family of $\varphi$-invariant automorphisms on $M$. Let $N$ be a von Neuman subalgebra with conditional expectation $\varepsilon$. For any $a \in \operatorname{Aut}_{\varphi}(M)$ the conditional expectation $\varepsilon_{a}$ onto $N_{a}=a(N)$ exists. Indeed $\varepsilon_{a}=a \circ \varepsilon \circ a^{-1}$. Let $\left\{a_{\lambda}\right\}$ $\subseteq \operatorname{Aut}_{\varphi}(M)$ be a net such that $a_{\lambda} \rightarrow a$ strongly as $\lambda \uparrow$ for some $a \in \operatorname{Aut}_{\varphi}(M)$. Then $\varepsilon_{a_{\lambda}}(x) \rightarrow \varepsilon_{a}(x)$ strongly as $\lambda \uparrow$ for every $x \in M$. Hence, by Theorem 2

$$
s-\liminf N_{a_{\lambda}}=w \text {-lim sup } N_{a_{\lambda}}=N_{a} \text {. }
$$

(iv) Let $M$ be the $2 \times 2$ matrix algebra, $\varphi$ the normalized trace on $M$, and $N$ the set of diagonal matrices in $M$. Define

$$
U_{n}=\left(\begin{array}{cc}
\cos \pi / n & \sin \pi / n \\
-\sin \pi / n & \cos \pi / n
\end{array}\right)
$$

and $N_{n}=U_{n}^{*} N U_{n}$ for $n=1,2, \ldots$ Then

$$
s-\lim \inf N_{n}=w-\lim \sup N_{n}=N,
$$

but $\mathrm{V}_{m} \bigcap_{n \geqslant m} N_{n}=\mathrm{C} \cdot 1$ and $\bigcap_{m} \mathrm{~V}_{n \geqslant m} N_{n}=M$.

(v) Let $N_{1}$ and $N_{2}$ be von Neumann subalgebras with conditional expectations, $S_{1}$ (resp. $S_{2}$ ) the unit balls of $N_{1}$ (resp. $N_{2}$ ), and $P_{1}$ (resp. $P_{2}$ ) the orthogonal projections onto $\overline{N_{1} \Phi}$ (resp. $\overline{N_{2} \Phi}$ ). Define

$$
d\left(N_{1}, N_{2}\right)=\max \left\{\sup _{x \in S_{1}}\left\|x \Phi-P_{2} x \Phi\right\|, \sup _{x \in S_{2}}\left\|x \Phi-P_{1} x \Phi\right\|\right\} .
$$

This is the so-called Hausdorff distance between $S_{1} \Phi$ and $S_{2} \Phi$. Suppose that there exists a von Neumann subalgebra $N$ with conditional expectation $\varepsilon$ such that $d\left(N_{\alpha}, N\right) \rightarrow 0$ as $\alpha \uparrow$. Let $P$ be the orthogonal projection onto $\overline{N \Phi}$. Then for any $\xi \in H$ with $\|\xi\| \leqslant 1$

$$
\begin{aligned}
\left\|P \xi-P_{\alpha} \xi\right\|^{2} & =\langle P \xi \mid \xi\rangle-\left\langle P_{\alpha} P \xi \mid \xi\right\rangle-\left\langle P P_{\alpha} \xi \mid \xi\right\rangle+\left\langle P_{\alpha} \xi \mid \xi\right\rangle \\
& \leqslant\left\|P \xi-P_{\alpha} P \xi\right\|+\left\|P P_{\alpha} \xi-P_{\alpha} \xi\right\| \\
& \leqslant 2 \cdot d\left(N_{\alpha}, N\right) \rightarrow 0 \quad(\text { as } \alpha \uparrow) .
\end{aligned}
$$

Therefore $P_{\alpha} \rightarrow P$ strongly, and by $2^{\circ}$ and $3^{\circ}$ in $\$ 1$ we have $\varepsilon_{\alpha}(x) \rightarrow \varepsilon(x)$ strongly for every $x \in H$. Thus, by Theorem 2

$$
s-\lim \inf N_{\alpha}=w-\lim \sup N_{\alpha}=N .
$$


The converse is not always true. Let $\left\{N_{\alpha}\right\}$ be strictly increasing and $N=\mathrm{V}_{\alpha} N_{\alpha}$. Then for any $\alpha$ there exists $\xi \in \overline{N \Phi}$ with $\|\xi\|=1$ and $\xi \perp \overline{N_{\alpha} \Phi}$. Hence $d\left(N_{\alpha}, N\right)=1$ for every $\alpha$, and $N_{\alpha}$ does not converge to $N$ in the Hausdorff metric topology, but

$$
s-\lim \inf N_{\alpha}=w-\lim \sup N_{\alpha}=N .
$$

3. Strong convergence of $\left\{\varepsilon_{\alpha}\right\}$. We now state our main theorem.

THEOREM 2. The following assertions are equivalent:

(i) $s-\lim \inf N_{\alpha}=w$-lim $\sup N_{\alpha}$;

(ii) there exists a conditional expectation $\varepsilon_{\infty}$ such that $\varepsilon_{\alpha}(x) \rightarrow \varepsilon_{\infty}(x)$ weakly for every $x \in M$;

(iii) $\left\{\varepsilon_{\alpha}(x)\right\}$ is a strongly convergent net for every $x \in M$;

(iv) $\left\{f \circ \varepsilon_{\alpha}\right\}$ is a convergent net in norm for every $f \in M_{*}$.

Moreover, if the above assertions are satisfied, then $\varepsilon_{\infty}$ in (ii) is the conditional expectation onto s-liminf $N_{\alpha}, \varepsilon_{\alpha}(x) \rightarrow \varepsilon_{\infty}(x)$ strongly, and $f \circ \varepsilon_{\alpha} \rightarrow f \circ \varepsilon_{\infty}$ in norm.

Proof. (i) $\Rightarrow$ (ii). Let $N_{\infty}=s$-liminf $N_{\alpha}$. The conditional expectation onto $N_{\infty}$ and the orthogonal projection onto $\overline{N_{\infty} \Phi}$ are denoted by $\varepsilon_{\infty}$ and $P_{\infty}$, respectively. Fix $x \in M$. Since $\left\{\varepsilon_{\alpha}(x)\right\}$ is uniformly bounded, for any subnet $\left\{\varepsilon_{\alpha^{\prime}}(x)\right\}$ of $\left\{\varepsilon_{\alpha}(x)\right\}$ there exists its subnet $\left\{\varepsilon_{\alpha^{\prime \prime}}(x)\right\}$ which converges to some $y \in M$ weakly. By the assumption $y \in N_{\infty}$. For any $z \in N_{\infty}$

$$
\begin{aligned}
\|x \Phi-y \Phi\| & \leqslant \liminf \left\|x \Phi-P_{\alpha^{\prime \prime}} x \Phi\right\| \\
& \leqslant \lim \left\|x \Phi-P_{\alpha^{\prime \prime}} z \Phi\right\|=\|x \Phi-z \Phi\|,
\end{aligned}
$$

because $P_{\alpha^{\prime \prime}} x \Phi \rightarrow y \Phi$ weakly and the norm of $H$ is weakly lower semicontinuous. Since $z \in N_{\infty}$ is arbitrary, we have $y \Phi=P_{\infty} x \Phi=\varepsilon_{\infty}(x) \Phi$. Thus $\varepsilon_{\alpha}(x) \rightarrow \varepsilon_{\infty}(x)$ weakly.

(ii) $\Rightarrow$ (iii). It follows from $2^{\circ}, 3^{\circ}$ and $4^{\circ}$ in $\S 1$.

(iii) $\Rightarrow$ (iv). Let $f \in M_{*}$ be fixed. It can be assumed without loss of generality that $f$ is positive. Then there exists $\xi \in H$ such that $f(x)=\langle\xi \mid x \xi\rangle$ for every $x \in M$ (see $[1$, Theorem 6]). For any $\alpha$ and $\beta$

$$
\begin{aligned}
\left\|f \circ \varepsilon_{\alpha}-f \circ \varepsilon_{\beta}\right\| & \leqslant \sup _{\|\cdot x\| \leqslant 1}\left|\left\langle\xi \mid \varepsilon_{\alpha}(x) \xi\right\rangle-\left\langle\xi \mid \varepsilon_{\beta}(x) \xi\right\rangle\right| \\
& \leqslant \sup _{\|\cdot x\| \leqslant 1}\left|\left\langle P_{\alpha} \xi \mid x \xi\right\rangle-\left\langle P_{\beta} \xi \mid x \xi\right\rangle\right| \\
& \leqslant\left\|P_{\alpha} \xi-P_{\beta} \xi\right\| \cdot\|\xi\| .
\end{aligned}
$$

Since $\left\{P_{\alpha} \xi\right\}$ is a Cauchy net, so is $\left\{f \circ \varepsilon_{\alpha}\right\}$ and we have (iv).

(iv) $\Rightarrow$ (i). Now we denote by $\varepsilon_{\alpha}^{*}$ the operator $f \mapsto f \circ \varepsilon_{\alpha}$ on $M_{*}$. Then $\varepsilon_{\alpha}^{*}$ is a norm-one projection (see [5]). Putting $\varepsilon_{\infty}^{*}(f)=s-\lim \varepsilon_{\alpha}^{*}(f)\left(f \in M_{*}\right), \varepsilon_{\infty}^{*}$ is a bounded linear operator on $M_{*}$. Furthermore, for any $\alpha$

$$
\begin{aligned}
\left\|\varepsilon_{\infty}^{*} \circ \varepsilon_{\infty}^{*}(f)-\varepsilon_{\infty}^{*}(f)\right\| \leqslant & \left\|\varepsilon_{\infty}^{*} \circ \varepsilon_{\infty}^{*}(f)-\varepsilon_{\alpha}^{*} \circ \varepsilon_{\infty}^{*}(f)\right\| \\
& +\left\|\varepsilon_{\alpha}^{* \circ} \varepsilon_{\infty}^{*}(f)-\varepsilon_{\alpha}^{*} \circ \varepsilon_{\alpha}^{*}(f)\right\|+\left\|\varepsilon_{\alpha}^{*}(f)-\varepsilon_{\infty}^{*}(f)\right\| \\
\leqslant & \left\|\varepsilon_{\infty}^{*} \circ \varepsilon_{\infty}^{*}(f)-\varepsilon_{\alpha}^{*} \circ \varepsilon_{\infty}^{*}(f)\right\|+2 \cdot\left\|\varepsilon_{\infty}^{*}(f)-\varepsilon_{\alpha}^{*}(f)\right\|,
\end{aligned}
$$


so that $\varepsilon_{\infty}^{*}$ is idempotent. We denote by $\varepsilon_{\infty}$ the conjugate operator of $\varepsilon_{\infty}^{*}$ on $M$. Then $\varepsilon_{\infty}(x)=w^{*}$-lim $\varepsilon_{\alpha}(x)$ for every $x \in M$ and $\varepsilon_{\infty}$ is also idempotent. We put $P_{\infty} x \Phi=$ $\varepsilon_{\infty}(x) \Phi(x \in M)$. Then $P_{\infty} x \Phi=w$-lim $P_{\alpha} x \Phi$ and

$$
\left\|P_{\infty} x \Phi\right\| \leqslant \liminf \left\|P_{\alpha} x \Phi\right\| \leqslant\|x \Phi\|
$$

for every $x \in M$. Hence $P_{\infty}$ is extended to a bounded linear operator on $H$. Since $\varepsilon_{\infty}$ is idempotent, so is $P_{\infty}$. Since

$$
\left\langle P_{\infty} x \Phi \mid y \Phi\right\rangle=\lim \left\langle P_{\alpha} x \Phi \mid y \Phi\right\rangle=\lim \left\langle x \Phi \mid P_{\alpha} y \Phi\right\rangle=\left\langle x \Phi \mid P_{\infty} y \Phi\right\rangle
$$

for every $x, y \in M, P_{\infty}$ is Hermitian. Therefore $P_{\infty}$ is an orthogonal projection on $H$. By $4^{\circ}$ in $\S 1$ we have $P_{\alpha} \rightarrow P_{\infty}$ strongly

Now fix $x \in w$-limsup $N_{\alpha}$. Then there exists a uniformly bounded net $\left\{x_{\alpha^{\prime}}\right\}$ such that $x_{\alpha^{\prime}} \in N_{\alpha^{\prime}}$ for each $\alpha^{\prime}$, where $\left\{N_{\alpha^{\prime}}\right\}$ is a subnet of $\left\{N_{\alpha}\right\}$, and $x_{\alpha^{\prime}} \rightarrow x$ weakly. For any $\xi \in H$

$$
\left\langle P_{\alpha^{\prime}} x \Phi-x_{\alpha^{\prime}} \Phi \mid \xi\right\rangle=\left\langle x \Phi-x_{\alpha^{\prime}} \Phi \mid P_{\alpha^{\prime}} \xi\right\rangle \rightarrow 0 \quad\left(\text { as } \alpha^{\prime} \uparrow\right),
$$

because $\left\{x \Phi-x_{\alpha^{\prime}} \Phi\right\}$ is uniformly bounded and $P_{\alpha^{\prime}} \rightarrow P_{\infty}$ strongly. Therefore, we have $P_{\infty} x \Phi=x \Phi$ and $\varepsilon_{\alpha}(x) \rightarrow x$ strongly. Thus $x \in s$-lim inf $N_{\alpha}$ and (i) is proved.

If a net $\left\{x_{\alpha}\right\}$ in $M$ satisfies that for any $\alpha$ there exists $y_{\alpha} \in M$ such that $x_{\beta}=\varepsilon_{\beta}\left(y_{\alpha}\right)$ for any $\beta \leqslant \alpha$, then $\left\{x_{\alpha}\right\}$ is called a martingale dominated by $\left\{y_{\alpha}\right\}$. A net $\left\{f_{\alpha}\right\}$ in $M_{*}$ is also called a martingale dominated by $\left\{g_{\alpha}\right\}$, if $f_{\beta}=g_{\alpha} \circ \varepsilon_{\beta}$ for any $\beta \preccurlyeq \alpha$.

THEOREM 3. (i) Let $\left\{x_{\alpha}\right\} \subseteq M$ be a martingale dominated by $\left\{y_{\alpha}\right\}$. If $\left\{y_{\alpha}\right\}$ is uniformly bounded, then there exists $x \in M$ such that $x_{\alpha}=\varepsilon_{\alpha}(x)$ for every $\alpha$.

(ii) Let $\left\{f_{\alpha}\right\} \subseteq M_{*}$ be a martingale dominated by $\left\{g_{\alpha}\right\}$. If $\left\{g_{\alpha}\right\}$ is weakly relatively compact, then there exists $f \in M_{*}$ such that $f_{\alpha}=f \circ \varepsilon_{\alpha}$ for every $\alpha$.

Proof. Since $\left\{y_{\alpha}\right\}$ is uniformly bounded, there exists a subnet $\left\{y_{\alpha^{\prime}}\right\}$ of $\left\{y_{\alpha}\right\}$ such that $y_{\alpha^{\prime}} \rightarrow x \sigma$-weakly for some $x \in M$. Because any conditional expectation is $\sigma$-weakly continuous, for any fixed $\alpha, \varepsilon_{\alpha}\left(y_{\alpha^{\prime}}\right) \rightarrow \varepsilon_{\alpha}(x) \sigma$-weakly as $\alpha^{\prime} \uparrow$. On the other hand, for sufficiently large $\alpha^{\prime}$ we have $\varepsilon_{\alpha}\left(y_{\alpha^{\prime}}\right)=x_{\alpha}$, so that $\varepsilon_{\alpha}(x)=x_{\alpha}$. Thus (i) is proved.

(ii) is also proved similarly, because $\psi \mapsto \psi \circ \varepsilon_{\alpha}$ is weakly continuous on $M_{*}$ for every $\alpha$.

The author would like to express his gratitude to Professor $\mathrm{H}$. Umegaki for his valuable advice and constant encouragement. The author also thanks the referee for his careful reading of this paper and kind suggestions.

\section{REFERENCES}

1. H. Araki, Some properties of modular conjugation operator of von Neumann algebras and a non-commutative Radon-Nikodym theorem with a chain rule, Pacific J. Math. 50 (1974), 309-354.

2. F. Hiai and M. Tsukada, Strong martingale convergence of generalized conditional expectations on von Neumann algebras, Trans. Amer. Math. Soc. 282 (1984), 791-798.

3. M. Takesaki, Conditional expectations in von Neumann algebras, J. Funct. Anal. 9 (1972), 306-321. 
4. M. Tsukada, Convergence of closed convex sets and $\sigma$-fields, Z. Wahrsch. Verw. Gebiete 62 (1983), $137-146$.

5. Strong convergence of martingales in von Neumann algebras, Proc. Amer. Math. Soc. 88 (1983), 537-540.

6. $301-309$.

7. H. Umegaki, Conditional expectation in an operator algebra, Tôhoku Math. J. (2) 6 (1954), 177-181.

8. __ Conditional expectation in an operator algebra. II, Tôhoku Math. J. (2) 8 (1956), 86-100.

Department of Information Sciences, Science University of Tokyo, Noda City, Chiba 278 , JAPAN 